\title{
Increased Calcification in Osteoprotegerin-Deficient Smooth Muscle Cells: Dependence on Receptor Activator of NF-KB Ligand and Interleukin 6
}

\author{
Andrea Callegari ${ }^{\mathrm{a}}$ Matthew L. Coons ${ }^{\mathrm{a}}$ Jerry L. Ricks ${ }^{\mathrm{b}}$ Michael E. Rosenfeld ${ }^{\mathrm{b}}$ \\ Marta Scatena ${ }^{a}$
}

Departments of ${ }^{\mathrm{a} B i o e n g i n e e r i n g}$ and ${ }^{\mathrm{b}}$ Pathology, University of Washington, Seattle, Wash., USA

\section{Key Words}

Osteoprotegerin - Receptor activator of NF-kappaB ligand · Interleukin 6 . Smooth muscle cells - Vascular calcification . Osteochondrogenic differentiation

\begin{abstract}
Objective: Vascular calcification is highly correlated with cardiovascular disease morbidity and mortality. Osteoprotegerin (OPG) is a secreted decoy receptor for receptor activator of NF-KB ligand (RANKL). Inactivation of OPG in apolipoprotein E-deficient (ApoE-/-) mice increases lesion size and calcification. The mechanism(s) by which OPG is atheroprotective and anticalcific have not been entirely determined. We investigated whether OPG-deficient vascular smooth muscle cells (VSMCs) are more susceptible to mineralization and whether RANKL mediates this process. Results: Lesionfree aortas from 12-week-old ApoE-/-OPG-/- mice had spotty calcification, an appearance of osteochondrogenic factors and a decrease of smooth muscle markers when compared to ApoE-/-OPG+/+ aortas. In osteogenic conditions, VSMCs isolated from ApoE-/-OPG-/- (KO-VSMC) mice deposited more calcium than VSMCs isolated from ApoE-/$\mathrm{OPG}+/+$ (WT-VSMC) mice. Gene expression and biochemical analysis indicated accelerated osteochondrogenic differentiation. Ablation of RANKL signaling in KO-VSMCs rescued
\end{abstract}

the accelerated calcification. While WT-VSMCs did not respond to RANKL treatment, KO-VSMCs responded with enhanced calcification and the upregulation of osteochondrogenic genes. RANKL strongly induced interleukin 6 (IL-6), which partially mediated RANKL-dependent calcification and gene expression in KO-VSMCs. Conclusions: OPG inhibits vascular calcification by regulating the procalcific effects of RANKL on VSMCs and is thus a possible target for therapeutic intervention.

(c) 2014 S. Karger AG, Basel

\section{Introduction}

Vascular calcification, a type of ectopic soft tissue mineralization, increases the risk of cardiovascular mortality. Arterial wall mineralization is estimated to be present in the vast majority of patients affected by cardiovascular disease (CVD) [1]. Patients with end-stage renal disease generally have extensive medial and intimal calcification that is associated with a highly significant increase in cardiovascular mortality compared to the general population [2]. Type II diabetics are an additional population affected by elevated vascular calcification [3]. Given the dramatic rise in diabetes and metabolic syndrome in the USA, an increase in cases of vascular calcification and CVD is to be expected in the coming decade.

\section{KARGER}

E-Mail karger@karger.com

www.karger.com/jvr (c) 2014 S. Karger AG, Base

$1018-1172 / 14 / 0512-0118 \$ 39.50 / 0$
Dr. Marta Scatena

Department of Bioengineering, University of Washington Box 358056

Seattle, WA 98195-8056 (USA)

E-Mail mscatena@uw.edu 
In the aorta, calcification promotes congestive heart failure by compromising vessel compliance and elasticity while in coronary and carotid arteries, calcium deposits may cause atherosclerotic plaque instability [1]. In human lesions, the process leading to vascular calcification appears to follow a pathway analogous to endochondral bone formation; however, in mouse lesions, vascular calcification is accompanied by the appearance of cartilaginous metaplasia that subsequently mineralizes $[4,5]$. In vitro and in vivo mechanistic studies indicate that vascular calcification is a highly regulated process involving vascular smooth muscle cells (VSMCs). Inorganic phosphate, bone morphogenetic protein (BMP) and oxidative-stress signaling have emerged as key regulators of osteochondrogenic transdifferentiation of VSMCs. Upregulation of the osteochondrogenic transcription factor Runx2 and other osteochondrogenic markers, and downregulation of SMC lineage markers appear to be key processes in vascular cell-dependent mineralization $[3,6,7]$.

Osteoprotegerin (OPG) is a member of the TNFreceptor superfamily and acts as a soluble receptor for receptor activator of NF- $\mathrm{BB}$ ligand (RANKL) and TNFrelated apoptosis-inducing ligand (TRAIL). Functional studies in vitro and in vivo indicate that OPG is a major regulator of bone remodeling by blocking RANKL binding to its own cell surface receptor RANK, and thus inhibiting RANKL-dependent osteoclast formation [8]. RANKL/RANK also modulates adaptive immunity [9, 10].

RANKL and OPG are present in atherosclerotic lesions in mice and humans; however, their roles in mediating vascular disease are not fully understood $[5,11,12]$. We previously reported that OPG deficiency in mice led to increased atherosclerosis, vascular calcification and osteoporosis that correlated with elevated circulating RANKL [13]. In addition, we have recently reported that in ApoE-/- mice, vessel wall-derived OPG protects from atherosclerosis and vascular calcification [14]. Treatment of LDLR-/- mice with recombinant OPG was found to reduce vascular calcification [15]. OPG is expressed by the endothelium in early human atherosclerotic lesions, in SMCs of fibrous cap and fibrocalcific lesions and it lines bone structure in advanced plaques. RANKL is mainly found in the extracellular matrix surrounding vascular calcium deposits [12]. Rattazzi et al. [5] first reported the expression of OPG and RANKL in lesions in the innominate arteries of ApoE-/- mice. In vitro, OPG and RANKL are expressed by VSMCs and endothelial cells and are regulated by inflammatory cytokines $[16$,
17]. In addition, several epidemiologic studies have linked OPG to the severity of CVD $[18,19]$. These findings point to an active role for OPG in the maintenance of cardiovascular homeostasis.

The increase in lesion size and vascular calcification occurs when the OPG gene is inactivated in ApoE-/mice either in the vessel wall or in the hematopoietic compartment; this strongly supports the notion that OPG is an atheroprotective molecule [13, 14]. However, the mechanisms by which OPG is atheroprotective and anticalcific are not known. For example, it is still unknown whether the absence of OPG in SMCs affects their response to calcification conditions and to RANKL. Thus, in this study, we tested the hypothesis that OPG-deficient VSMCs mineralize more than WT VSMCs and that this is dependent on unopposed RANKL signaling.

\section{Material and Methods}

Animals

Generation of ApoE-/-OPG-/- mice was described in Bennett et al. [13]. Animals were maintained in a specific pathogen-free environment and fed a normal chow diet. All protocols are in compliance with the NIH Guideline for the Care and Use of Laboratory Animals and were approved by the University of Washington Institutional Animal Care and Use Committee.

Tissue Preparation, Histochemical and Immunohistochemical Staining

Innominate arteries from 12-week-old ApoE-/-OPG-/- and ApoE-/-OPG $+/+$ mice were fixed in formalin and embedded in paraffin. Sections (5- $\mu$ m-thick) were used for histochemical and immunohistochemical analyses. Alizarin Red S or von Kossa staining was used to detect calcium deposition. The following antibodies were employed: anti-goat SMa-actin (Sigma, St. Louis, Mo., USA), goat anti-SM22a (Abcam, Cambridge, Mass., USA), rabbit anti-phosphorylated ERK (CellSignaling, Danvers, Mass., USA), goat anti-Runx2 (R\&D Systems, Minneapolis, Minn., USA), mouse anti-RANKL (Imgenex, San Diego, Calif., USA), goat antiosteopontin (R\&D Systems), rabbit anti-cleaved caspase-3 (CellSignaling), control rabbit, goat and mouse IgG (Invitrogen, Carlsbad, Calif., USA), biotin-conjugated rabbit anti-goat IgG (Pierce Thermoscientific, Rockford, Ill., USA), biotin-conjugated goat anti-rabbit IgG (Invitrogen) and biotin-conjugated rabbit antimouse IgG (Invitrogen). Sections were counterstained with hematoxylin (Ricca Chemical Company, Arlington, Tex., USA) or methyl green (Sigma).

\section{VSMC Isolation and Characterization}

Aortas from wild-type, OPG-/-, ApoE-/-OPG-/- and ApoE-/-OPG+/+ mice (6-10 weeks old) were incubated in an enzyme-mix solution containing $2 \mathrm{mg} / \mathrm{ml} \mathrm{BSA}$ (Sigma), $1 \mathrm{mg} / \mathrm{ml} \mathrm{col-}$ lagenase CLS-1 (Worthington, Freehold, N.J., USA), $0.375 \mathrm{mg} / \mathrm{ml}$ soybean trypsin inhibitor (Worthington), $0.125 \mathrm{mg} / \mathrm{ml}$ elastase type III (Sigma) for $5 \mathrm{~min}$ at $37^{\circ} \mathrm{C}$. The adventitia was then re- 
moved, the endothelium stripped and the media cut into small pieces that were dispersed in a mixture of $0.6 \mathrm{mg} / \mathrm{ml}$ collagenase CLS-2 (Worthington) and $0.25 \mathrm{mg} / \mathrm{ml}$ elastase type III (Sigma) in culture medium containing FBS and incubated at $37^{\circ} \mathrm{C}$ for $1 \mathrm{~h}$. The cell suspension was centrifuged and resuspended in DMEM culture medium (Invitrogen) containing $100 \mathrm{U} / \mathrm{ml}$ penicillin, $100 \mathrm{mg} /$ $\mathrm{ml}$ streptomycin and $20 \%$ FBS. The cells were split when confluent and cultured in growth media with $10 \%$ FBS after passage 3 . The cells used for the experiments were primary cultures and subcultures of 3-9 passages.

Cells at passage 1 were plated on glass chamber slides (Lab-Tek, Rockford, Ill., USA) and used for cell characterization by immunofluorescence. Cells were stained with antibodies against SMaactin (Sigma), SM22a (Abcam), desmin (Dako, Carpinteria, Calif., USA) and CD31 (BD Bioscience, San Jose, Calif., USA). Alexa fluor 594 goat anti-mouse, alexa fluor 594 goat anti-rabbit and alexa fluor 594 rabbit anti-goat (Invitrogen) were used as secondary antibodies.

\section{Retroviral Transduction of $O P G$}

OPG cDNA (a gift from Dr. Ed Clark, University of Washington) was cloned into the $\mathrm{PBMN}-\mathrm{I}-\mathrm{GFP}$ retroviral vector and retroviral particles were generated as described by Rice et al. [20]. The pBMN-I-GFP retroviral particles were used as a control. After infection, the cells were sorted by FACS for GFP.

\section{RNA Silencing}

To degrade complementary messenger RNA, siRNAs for RANKL (Ambion Silencer Select Pre-designed s75266, Carlsbad, Calif., USA) were utilized. The SMCs were transfected with the Amaxa Basic Nucleofector kit for primary mammalian SMCs (Lonza, Switzerland) following the manufacturer's protocol. Gene knockdown was confirmed by verifying mRNA levels by qPCR.

\section{Calcification Assay}

VSMCs were plated at a density of $7,000 \mathrm{cell} / \mathrm{cm}^{2}$ in 6 -well plates and cultured in control medium (CM, i.e. DMEM/3\% FBS) or in osteogenic medium (OM, i.e DMEM/3\% FBS and $2.6 \mathrm{mM}$ inorganic phosphate). The media were changed every 2 days. The cells were harvested after 2 and 4 days for RNA, and the calcium assay was performed after 7 days. Recombinant mouse RANKL (R\&D Systems) was used at $100 \mathrm{ng} / \mathrm{ml}$ and anti-IL-6 antibody (eBioscience, San Diego, Calif., USA) or rat IgG1k control were added to the culture media at $0.1 \mu \mathrm{g} / \mathrm{ml}$.

\section{Calcium Assay}

Cell cultures were rinsed with $\mathrm{PBS} 0.6 \mathrm{~mol} / \mathrm{l} \mathrm{HCl}$ at $4{ }^{\circ} \mathrm{C}$ for $24 \mathrm{~h}$. Calcium released from the cells was determined colorimetrically by the o-cresolphthalein complexone method as described previously (TECO calcium diagnostic kit, Anaheim, Calif., USA) [21]. Calcium was normalized to cellular protein and expressed as $\mu \mathrm{g} / \mathrm{mg}$ protein.

\section{Alkaline Phosphatase Activity}

For determination of cellular alkaline phosphatase (ALP) activity, cells were washed 3 times with PBS and assayed for ALP activity using the ALP colorimetric assay kit (Biovision, Milpitas, Calif., USA) according to the manufacturer's protocol. ALP activity was normalized to total cellular protein concentration and expressed as $\mathrm{U} / \mathrm{mg}$ protein.

\section{Real-Time Quantitative PCR}

Total RNA was isolated from the aortas of 12-week-old ApoE-/-OPG-/- or ApoE-/-OPG+/+ mice following removal of the adventitia and endothelium and from the cultured VSMCs using the RNeasy kit (Qiagen, Germantown, Md., USA). cDNA synthesis was performed using RevertAid First Strand cDNA synthesis kit (Fermentas, Rockford, Ill., USA). mRNA levels were quantified by Taqman real-time PCR and the ABI Prism 7500 (Applied Biosystems, Carlsbad, Calif., USA). The following gene expression assays (Invitrogen) were used:

Spp1 (OPN) Mm00436767_m1;

MGP Mm00485009_m1;

BMP2 Mm01340178_m1;

Runx2 Mm00501584_m1;

IL-6 Mm00446190_m1;

SMa-actin Mm01546133_m1;

SM22a Mm00441660_m1;

ALP Mm00475834_m1.

Quantification of gene expression was calculated by the standard curve method as described in the Invitrogen manual (http:// www.icmb.utexas.edu/core/DNA/Information_Sheets/Realtime\%20PCR/Guide_to_Relative_Quantitation.pdf). Briefly, $1 \mu \mathrm{g}$ cDNA from calvaria or VSMCs was diluted to $1,0.5$ and 0.25 to create an appropriate standard curve for each different Taqman primers-probe set. The curves were then used to quantitate the mRNA amount for each gene from the treated groups. Data were normalized to the $18 \mathrm{~S}$ rRNA.

\section{Reverse Transcriptase PCR}

cDNA from tissue and cells lysates was also utilized in reverse transcriptase PCR using GoTaq DNA polymerase (Promega, Madison, Wisc., USA). cDNA from calvaria cells served as a positive control. The PCR primers for mouse RANK were: forward 5'-AGATGTGGTCTGCAGCTCTTCCAT-3' , and reverse $5^{\prime}$-AC ACACTTCTTGCTGACTGGAGGT-3', amplifying a 278-bp fragment. Primers for RANKL were forward $5^{\prime}$-CGCTCTGTTCCT GTACTTTCGAGCG-3' and reverse $5^{\prime}$-TCGTGCTCCCTCCT TTCATCACAGGTT-3', amplifying a 198-bp fragment. Primers for GAPDH were: forward $5^{\prime}$-CAAGGTCATCCATGACAACT TTG-3', and reverse 5'-GTCCACCACCCTGTTGCTGTAG-3', amplifying a 496-bp fragment.

\section{Western Blot Analysis}

Lysates were produced from VSMC cultures and diluted with Laemmli buffer [62.5 mM TRIS-HCl pH 6.8, 25\% (w/v) glycerol, $2 \%(\mathrm{w} / \mathrm{v})$ SDS and $0.01 \%(\mathrm{w} / \mathrm{v})$ bromophenol blue]. Protein concentrations were determined using a Micro BCA protein assay (Pierce Thermoscientific). Equal quantities of protein were loaded into each well of a mini-PROTEAN TGX gel (Bio-Rad) and SDSPAGE was performed using the mini-PROTEAN tetra system (Bio-Rad). Sample proteins were then transferred to an immunoblot PVDF membrane (Bio-Rad) and detected using proteinspecific antibodies, horseradish peroxidase-conjugated antibodies and Pierce ECL Western Blotting Substrate (Pierce Thermoscientific).

The following antibodies were utilized: anti-phosphop44/42 MAPK (ERK1/2) 1:2,000 (CellSignaling), anti-p44/42 MAPK (ERK1/2) 1:1,000 (CellSignaling), anti-beta actin as loading control 1:1,000 (Abcam), anti-SMa-actin 1:1,000 (Dako), anti-RANKL (R\&D Systems), anti-rabbit IgG peroxi- 
dase conjugate (Sigma), anti-goat IgG HRP (Santa Cruz Technologies, Santa Cruz, Calif., USA) and anti-mouse IgG peroxidase (Sigma).

\section{ELISA Assay}

Conditioned media from the treated VSMCs were collected for measurement of OPG (ELISA kit from R\&D Systems) and IL-6 (kit from eBioscience) according to the manufacturer's instructions.

\section{Luciferase Reporter Assay}

To study the activity of the transcription factor Runx2, a dualluciferase reporter assay system (Promega, Rockford, Ill., USA) was used. Briefly, VSMCs were transiently transfected using the liposome-mediated method (Lipofectamine 2000, Invitrogen) with p6OSE2 containing the firefly luciferase gene downstream of Runx2 response elements [22]. Promoter-less pGL4.10 vector served as a background control. The plasmid pRL containing the Renilla luciferase reporter gene was cotransfected to normalize for transfection efficiency. We found that transfection with Lipofectamine 2000 routinely yielded 5-10\% transfected cells. The cells were lysed and luciferase activity was measured $72 \mathrm{~h}$ after transfection according to the manufacturer's protocol.

\section{Statistical Analysis}

Data are expressed as mean \pm SE. Significant differences between groups was determined by the Student t test or ANOVA. Data were considered statistically significant at $\mathrm{p}<0.05$.

\section{Results}

Altered Expression of Calcification Regulatory Factors in ApoE-/-OPG-/- Mouse Aortas

We have previously shown that ApoE-/-OPG-/- mice present more vascular calcification than ApoE-/-OPG $+/+$ at 40 and 60 weeks of age [13]. Here, we asked whether calcification-related genes were dysregulated in young, 12-week-old, ApoE-/-OPG-/- mouse aortas when compared to those of age-matched ApoE-/-OPG+/+ mice. We performed qPCR expression analysis on whole mouse aortas isolated from young, nondiseased mice and found decreased MGP expression and increased OPN expression in the aortas from the ApoE-/-OPG-/- mice compared to the ApoE-/-OPG+/+ mice (fig. 1a, b). We also found sporadic medial vascular calcification in ApoE-/OPG-/- aortas but not in ApoE-/-OPG+/+ aortas by Alizarin Red S staining (2/6 ApoE-/-OPG-/- had calcification and 0/6 ApoE-/-OPG+/+ had calcification; fig. 1c, d; online suppl. fig. 1; for all online material, see www. karger.com/doi/10.1159/000358920). However, statistical significance was not reached at this early age as previously shown [13]. Histological staining showed expression of OPN, Runx2 and phosphorylated ERK associated with early medial calcification in ApoE-/-OPG-/- but not in
ApoE-/-OPG+/+ aortas (fig. 1c; online suppl. fig. 1). Further, in the areas of early calcification, SMa-actin and SM22a had disappeared (fig. 1d). There was no sign of cell death in these regions as determined by active caspase 3 staining (online suppl. fig. 1). These data suggest that in the absence of OPG, unabated OPG-ligand signaling may lead to accelerated osteochondrogenic conversion.

\section{KO-VSMCs Have Enhanced Calcification in Response} to Osteogenic Conditions

We then reasoned that VSMCs isolated from ApoE-/OPG-/- (KO-VSMCs) and ApoE-/-OPG+/+ (WTVSMCs) may show accelerated mineralization in response to OM. Indeed, OPG expression has been shown to decrease in response to osteogenic conditions in VSMCs [23], findings that we have confirmed in our WT-VSMCs (not shown). We found that freshly isolated (passage 1) KO-VSMCs expressed the smooth muscle cell markers desmin, SMa-actin and SM22 $\alpha$ as expected and similar to WT-VSMCs (fig. 2a); however, when cultured in OM for 7 days, KO-VSMCs showed increased calcification and increased ALP activity as compared to WT-VSMCs (fig. 2b; online suppl. fig. 2). WT-VSMCs secreted very high levels of OPG (fig. 2c). Taken together, these data suggest that unabated signaling initiated by OPG ligands may drive the increased calcification observed in $\mathrm{KO}$ VSMCs. Similarly, we found that OPG-/- VSMCs calcified more when compared to OPG+/+ VSMCs, thus indicating that expression of the ApoE gene does not affect VSMC calcification (online suppl. fig. 3).

\section{KO-VSMCs Have an Enhanced Osteochondrogenic Phenotype}

We further asked whether KO-VSMCs have altered expression of osteochondrogenic genes similarly to what was observed in vivo in the aortas. By using $\mathrm{qPCR}$, we found that in OM, OPN was more strongly induced in KO-VSMCs than in WT-VSMCs, and that MGP was repressed more in KO-VSMCs compared to WT-VSMCs (fig. 3a, b). Further, KO-VSMCs expressed lower levels of SMa-actin as compared to the WT-VSMCs when cultured for 4 days in CM and OM (fig. 3e). Similarly, we found that Runx2 promoter activity was higher in the KO-VSMCs than in WT-VSMCs (fig. 3c), which correlated with elevated ERK phosphorylation in the KOVSMCs cultured in CM and in OM (fig. 3d). These data suggest that a lack of OPG may affect the expression of SMa-actin and activation of the ERK-Runx2 pathway, even in control conditions $[22,24]$. Treatment with OM did not appear to reduce SMa-actin further in $\mathrm{KO}$ - 
Fig. 1. Altered expression of calcificationregulatory factors in mouse aortas. a, b qPCR of RNA from aortic tissue (ascending, arch and thoracic) isolated from ApoE-/-OPG-/- mice $(\mathrm{n}=12)$ and ApoE-/-OPG $+/+(\mathrm{n}=6)$ at 12 weeks of age. a MGP. b OPN. * $\mathrm{p}<0.05$. c, d Immunohistochemistry of ApoE-/-OPG-/- innominate artery serial sections for phosphorylated ERK, Runx2, OPN, SM22a, $\mathrm{SM} \alpha$-actin, control goat, rabbit and mouse IgG and for Alizarin Red.



VSMCs. Further, caspase-dependent cell death did not mediate the increased mineralization of KO-VSMCs, determined by incubation with the caspase inhibitor zVAD (online suppl. fig. 4). Taken together, these data suggest that the increased KO-VSMC calcification is likely regulated by accelerated osteochondrogenic transition rather than by increased cell death.
Inhibition of RANKL Signaling in KO-VSMCs Rescues the Increased Calcification

We then reasoned that re-expression of OPG may rescue the calcification phenotype and gene expression observed in KO-VSMCs. We found that transgenic KOVSMCs ( ${ }^{\mathrm{TG}} \mathrm{KO}-\mathrm{VSMCs}$ ) in which OPG was retrovirally reintroduced, calcified significantly less than vector 

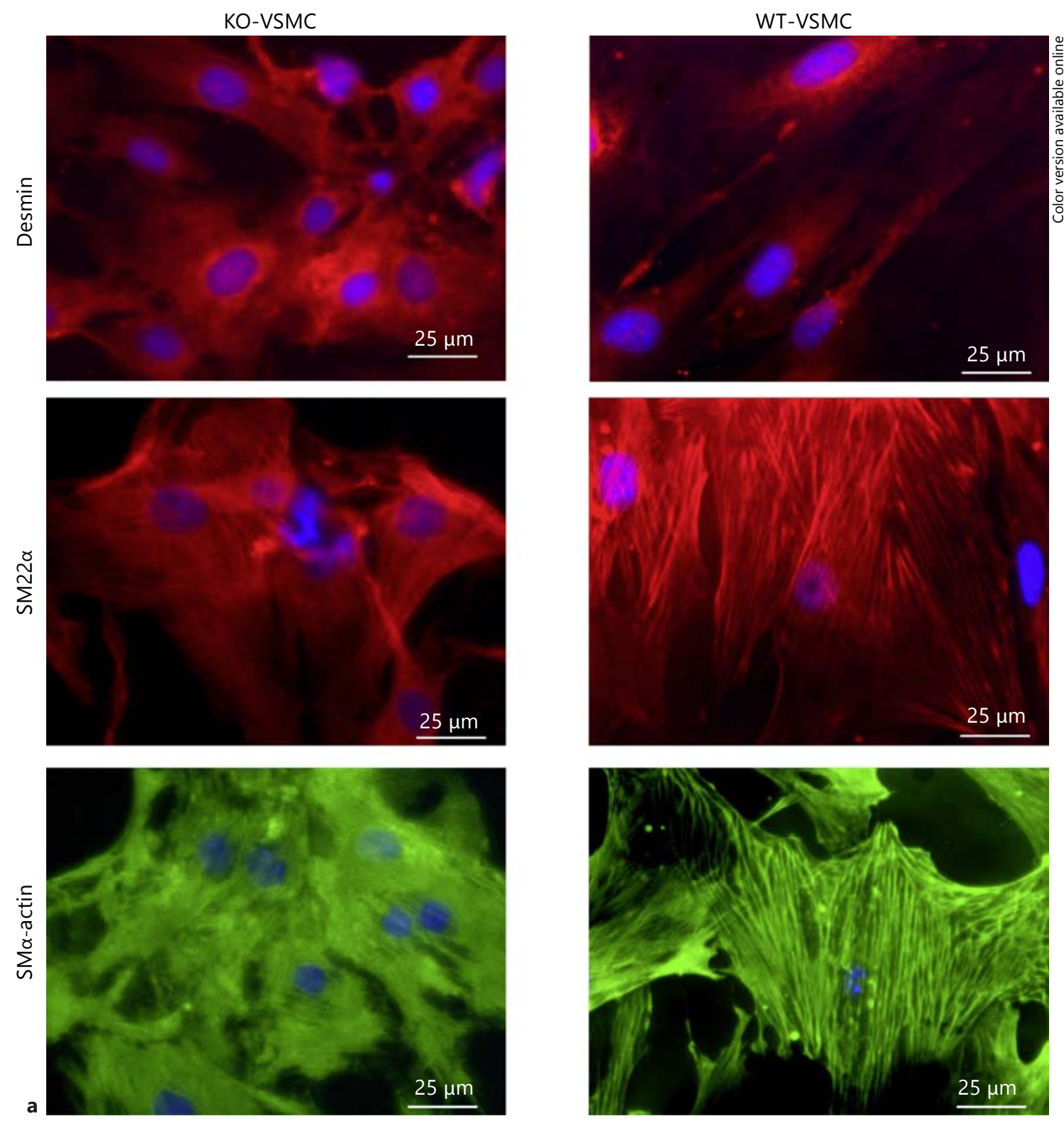

Fig. 2. Characterization of freshly isolated KO-VSMCs and WT-VSMCs. a Immunofluorescence for desmin, SM22 $\alpha$, SM $\alpha$-actin in KO-VSMCs and WT-VSMCs one passage after isolation.

control KO-VSMCs ( ${ }^{\mathrm{GPF}} \mathrm{KO}-\mathrm{VSMCs}$ ) which were retrovirally infected with the control vector expressing GFP (fig. 4a). As expected, ${ }^{\mathrm{TG}} \mathrm{KO}-\mathrm{VSMCs}$ produced $\mathrm{OPG}$ (online suppl. fig. 5A). Further, ${ }^{\mathrm{TG}} \mathrm{KO}-\mathrm{VSMCs}$ expressed lower levels of OPN and higher levels of MGP mRNA than GPFKO-VSMCs, again suggesting that unabated OPG ligands may be responsible for the accelerated osteochondrogenic differentiation (online suppl. fig. 5B, C).

Calcification in OPG-Deficient VSMCs
We further hypothesized that RANKL could be responsible for the accelerated calcification of KO-VSMCs. We first confirmed that RANKL and RANK were expressed by VSMCs qualitatively by RT-PCR (fig. 4b). We then asked whether blocking RANKL signaling by downregulating the receptor RANK with siRNA would rescue the calcification phenotype. RANK siRNA-transduced KO-VSMCs deposited less calcium when compared with scrambled siRNA (fig. 4c). As a control, we verified that

J Vasc Res 2014;51:118-131 


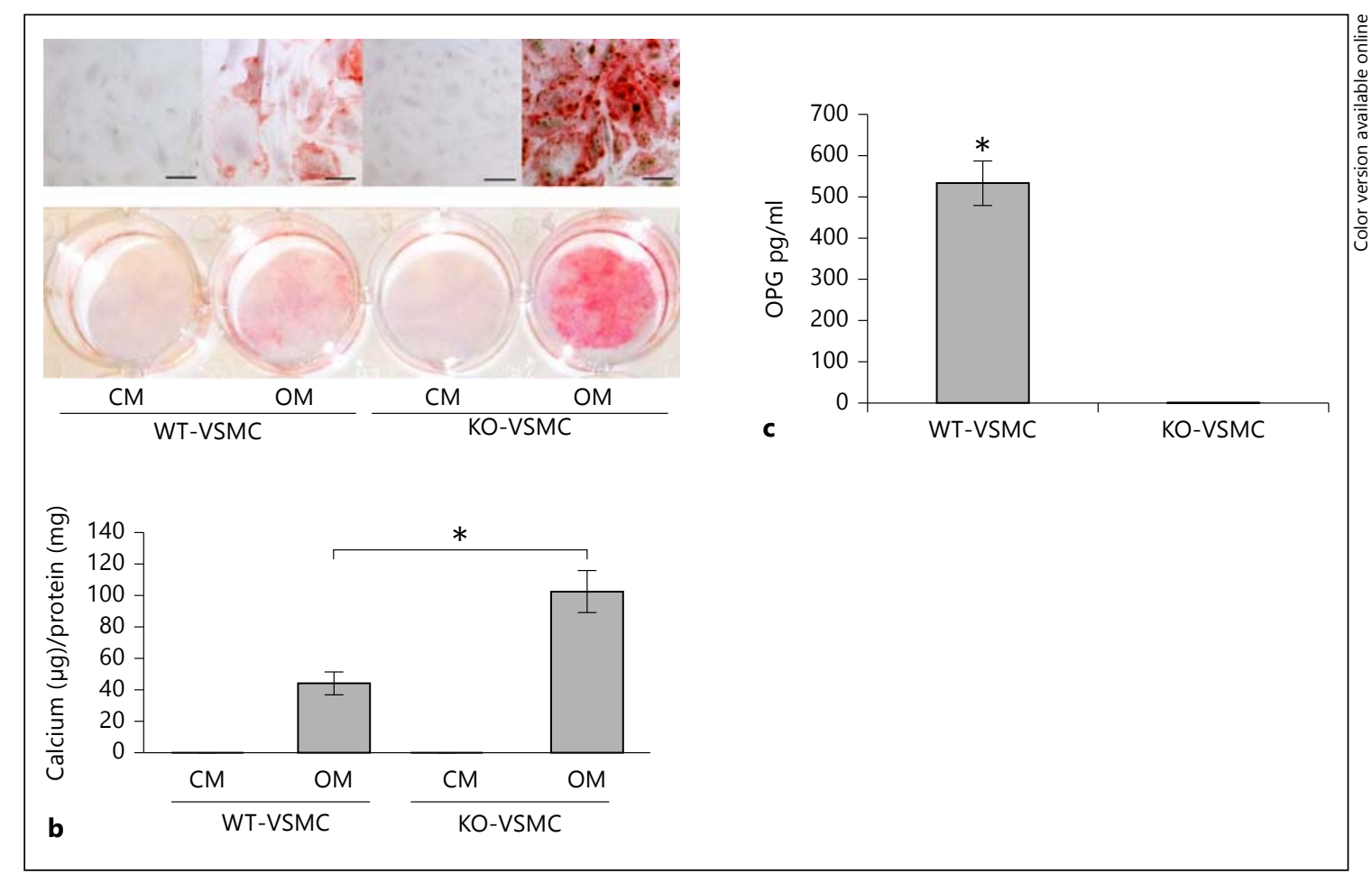

Fig. 2. Characterization of freshly isolated KO-VSMCs and WTVSMCs. b Calcification assay with WT-VSMCs and KO-VSMCs. Cells were cultured in $\mathrm{CM}$ and $\mathrm{OM}$ for 7 days. Calcium deposition was measured as described in Methods. ${ }^{*} \mathrm{p}<0.05$. Alizarin Red $S$ staining was also performed directly in the plate wells. Magnifications: low (bottom) and high (top). Scale bar $=50 \mu \mathrm{m}$. c ELISA for OPG on supernatant of WT-VSMCs and KO-VSMCs. ${ }^{*} \mathrm{p}<0.05$.
siRNA reduced RANK expression (online suppl. fig. 6). These data strongly implicate RANKL and RANK signaling in the enhanced calcification phenotype of $\mathrm{KO}$ VSMCs.

\section{RANKL Treatment Increases Calcification in KO-VSMCs}

We then asked whether the addition of exogenous RANKL in a dose-dependent manner would affect VSMC calcification. We found that RANKL dose-dependently enhanced KO-VSMC OM-dependent calcification (fig. 5a); however, RANKL had no effect on WT-VSMC calcification (fig. 5b). In KO-VSMCs, RANKL was able to induce activation as well as increase the expression of Runx2 (fig. 5c; online suppl. fig. 7). Further, in KOVSMCs, RANKL also upregulated OPN, BMP2 and ALP expression and downregulated MGP, SMa-actin and SM22a expression (online suppl. fig. 7).

These findings suggest that unopposed RANKL may accelerate osteochondrogenic differentiation in vitro as well in vivo. Indeed, we found that RANKL was expressed in ApoE-/- aortas regardless of genotype by RT-PCR.
Further, RANKL protein associated with calcified areas in young ApoE-/-OPG-/-mice innominate arteries, and it was also expressed in cells surrounding the mineral (fig. 5d: qualitative RT-PCR, 5e: immunohistochemistry). However, RANKL staining in ApoE-/-OPG+/+ innominate arteries appeared weaker that in ApoE-/OPG-/- arteries (fig. 5f).

\section{IL-6 Partially Mediates RANKL Enhancement of KO-VSMC Calcification}

We have recently shown that RANKL upregulates expression of IL- 6 and TNF- $\alpha$ in macrophages [25]. In this study, we determined that RANKL also induced IL-6 in KO-VSMCs and to a lesser extent in WT-VSMCs, measured by ELISA and independent of osteogenic conditions (fig. 6a). Similarly, IL-6 was upregulated in ApoE-/OPG-/- aortas compared to ApoE-/-OPG+/+ (fig. 6b) and downregulated in ${ }^{\mathrm{TG}} \mathrm{KO}-\mathrm{VSMCs}$ (not shown). IL-6 has recently been shown to be a promoter of vascular calcification [26]. Using neutralizing antibodies to IL-6 in combination with RANKL treatment, we observed that a portion of the RANKL-enhanced calcification was de- 


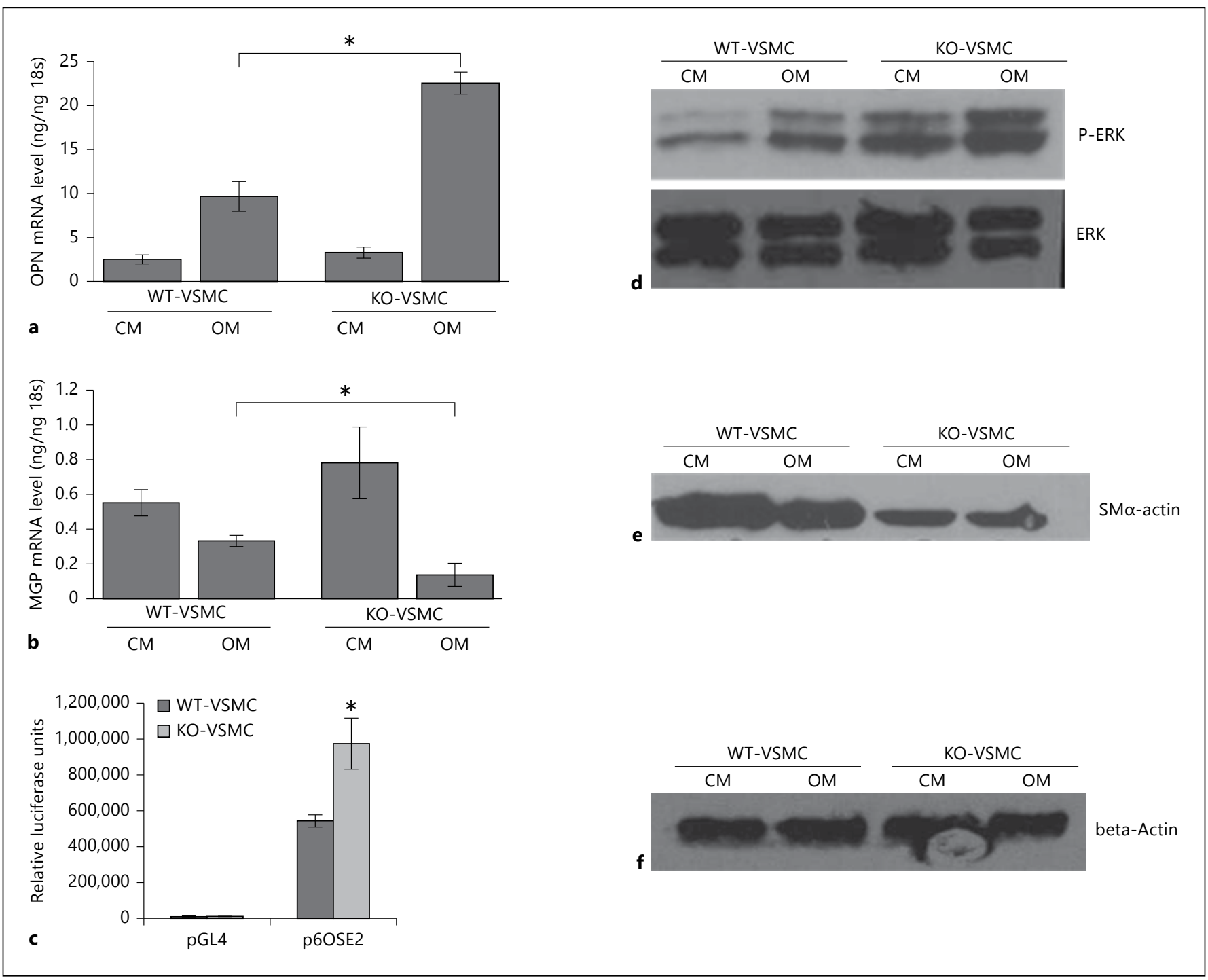

Fig. 3. Differential expression of regulatory factors in KO-VSMCs vs. WT-VSMCs. a, b Gene expression analysis between WTVSMCs and KO-VSMCs after 4 days treatment with CM and OM showed a higher level of OPN (a) and a lower level of MGP (b) in KO-VSMCs compared to WT-VSMCs in OM. Data are collected from 3 independent experiments. ${ }^{*} \mathrm{p}<0.05$. c Dual-luciferase reporter assay indicating that Runx 2 activity is higher in KO-VSMCs than in control WT-VSMCs. Cells were transduced with p6OSE2

pendent on IL-6 (fig. 6c). IL-6 by itself was a weak inducer of KO-VSMC calcification (online suppl. fig. 8). We further found that inhibition of IL- 6 reversed the regulation of a portion of the RANKL-dependent osteochondrogenic genes such as OPN, Runx2, BMP2, but not MGP (fig. 6d). Finally, we found that IL-6 is a strong inducer of RANKL, RANK and OPG, suggesting a possible forward loop (online suppl. fig. 8).

Calcification in OPG-Deficient VSMCs and pGL4 as described in Methods and cultured $72 \mathrm{~h}$ before harvesting for luciferase measurement. ${ }^{*} \mathrm{p}<0.05$ vs. p6OSE2-WTVSMC. d Western blot for phosphorylated ERK (P-ERK, top panel) and ERK (bottom panel) showing increased ERK activity in KO-VSMCs and increased ERK activity in OM regardless of cell genotype. e KO-VSMCs express less SMa-actin than WT-VSMCs. f beta-Actin was used as a loading control.

\section{Discussion}

Vascular calcification, a type of ectopic soft tissue mineralization, increases the risk of cardiovascular mortality [1]. We showed previously that vascular calcification is increased in ApoE-/- mice lacking OPG [13]. In addition, we recently showed that vessel wall-derived OPG protects ApoE-/- mice from the development of vascular 
Fig. 4. RANKL signaling inhibition in KOVSMCs rescues increased calcification. a Calcification assay with ${ }^{\mathrm{TG}} \mathrm{KO}-\mathrm{VSMCs}$, in which OPG was retrovirally reintroduced. ${ }^{\mathrm{TG}} \mathrm{KO}-\mathrm{VSMCs}$ calcified more than ${ }^{\mathrm{GPF}} \mathrm{KO}$-VSMCs, which were retrovirally infected with the control vector expressing GFP. ${ }^{\mathrm{TG}} \mathrm{KO}-\mathrm{VSMCs}$ and ${ }^{\mathrm{GFP}} \mathrm{KO}-\mathrm{VSMCs}$ were treated with $\mathrm{OM}$ for 7 days. ${ }^{*} \mathrm{p}<0.05$. b RT-PCR expression of RANK and RANKL in KO-VSMCs and in positivecontrol calvaria cells. c Calcification assay with scrambled transduced (Scramble) or RANK siRNA-transduced (RANK siRNA) KO-VSMCs. Cells were treated with CM or OM for 7 days. ${ }^{*} \mathrm{p}<0.05$.


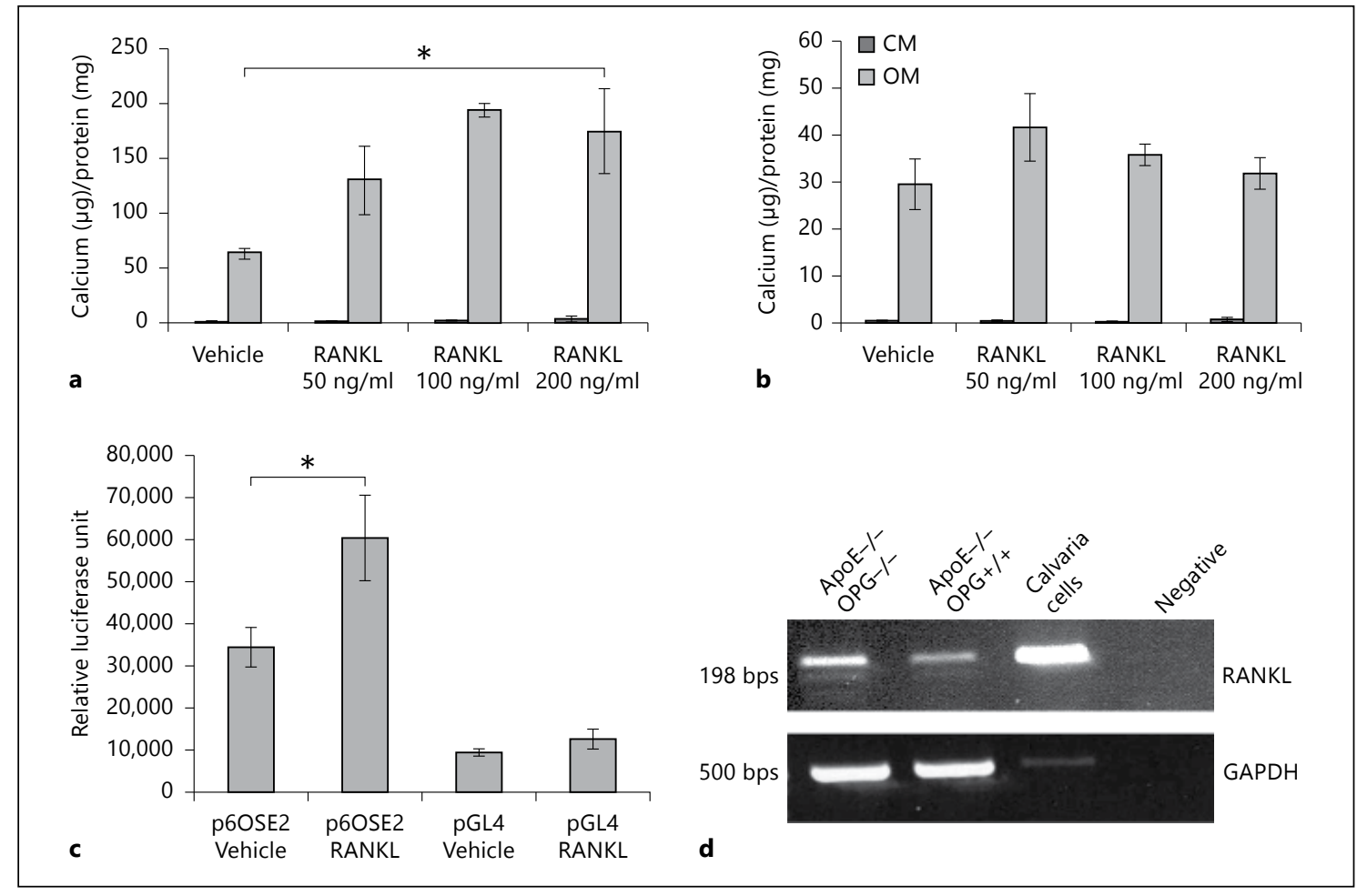

Fig. 5. RANKL enhanced calcification of KO-VSMCs but not of WT-VSMCs. KO-VSMCs (a) and WT-VSMCs (b) were treated with RANKL at 50, 100, $200 \mathrm{ng} / \mathrm{ml}$ for 7 days. ${ }^{*} \mathrm{p}<0.05$ RANKL all concentrations vs. vehicle. c Dual-luciferase reporter assay showing Runx2 activity increased in KO-VSMCs after treatment with RANKL for $72 \mathrm{~h}$. Cells were transduced with p6OSE2 and
pGL4 as described in methods and were cultured with vehicle or $100 \mathrm{ng} / \mathrm{ml}$ of RANKL before harvesting for luciferase measurement. ${ }^{*} \mathrm{p}<0.05$ p6OSE RANKL vs. p6OSE2. d RT-PCR for RANKL expression in freshly isolated aortas from 12-week-old ApoE-/OPG-/- and ApoE-/-OPG+/+ mice. Calvaria cells were used as a positive control. 



Fig. 5. RANKL enhanced calcification of KO-VSMCs but not of WT-VSMCs. e Staining for RANKL and von Kossa of 12-week-old ApoE-/-OPG-/- innominate artery. Left: the top panel is a wide view showing RANKL expression in medial cells closely associated with areas of calcification, in medial cells further from the calcified area and in few adventitial cells (arrows) and the bottom panel shows a higher magnification of RANKL staining associated with

calcification [14]. In this report, we sought to determine whether the lack of OPG specifically in VSMCs facilitated their conversion to a phenotype that is more prone to mineralization. We observed that KO-VSMCs deposited more calcium than WT-VSMCs, and that they expressed osteochondrogenic markers in a RANKL-dependent manner when cultured in osteogenic conditions. Taken together, these data strongly suggest that OPG inhibits VSMC calcification by neutralizing the promineralizing effects of RANKL.

There is increasing clinical evidence suggesting that OPG and RANKL may play an important role in CVD $[18,27]$. Several studies have shown that baseline serum RANKL is a highly significant predictor of CVD risk (including ischemic stroke, transient ischemic attack, myocardial infarction and vascular death) [28-30]. Moreover, there is a correlation between plasma RANKL and elevated circulating phosphate levels seen in chronic kidney disease patients, a population that is affected by extensive medial and intimal calcification and significant increases in cardiovascular mortality when compared to the general population [31]. Clinical evidence also links serum the calcified area (arrowhead). Middle: the top panel is a wide view showing von Kossa staining in the medial layer and the bottom panel shows a higher magnification of von Kossa staining (arrowhead indicates von Kossa staining intimately associated with RANKL-positive area). Right: this panel is a wide whole-vessel view of von Kossa staining. f RANKL staining on 12-week-old ApoE-/-OPG+/+ innominate artery.

OPG levels to CVD. The results of several studies have suggested that OPG is an additional prognostic biomarker of CVD and mortality in high-risk populations, along with age, diabetes, markers of systemic inflammation, chronic infection and smoking [18, 32-34]. It is being debated whether elevated serum OPG is causative or a compensatory protective response to elevated levels of RANKL in people with CVD.

In animal models, OPG appears to be a vascular protective factor. As noted, we have shown that a lack of OPG in ApoE-/- mice results in larger lesions and increased mineralization of the vasculature, and we recently determined that either vessel wall-derived or bone marrow-derived OPG protected against atherosclerosis and vascular calcification in ApoE-/- mice [13, 14]. Furthermore, administration of OPG to fat fed atheroprone LDLR-/- mice leads to less mineral accumulation in the vasculature [15]. With this study, we also show that in the absence of OPG, microcalcification accompanied by expression of osteochondrogenic factors and disappearance of smooth muscle markers occurs in the medial layer of lesion-free young ApoE-/-OPG-/- mice (fig. 1), 


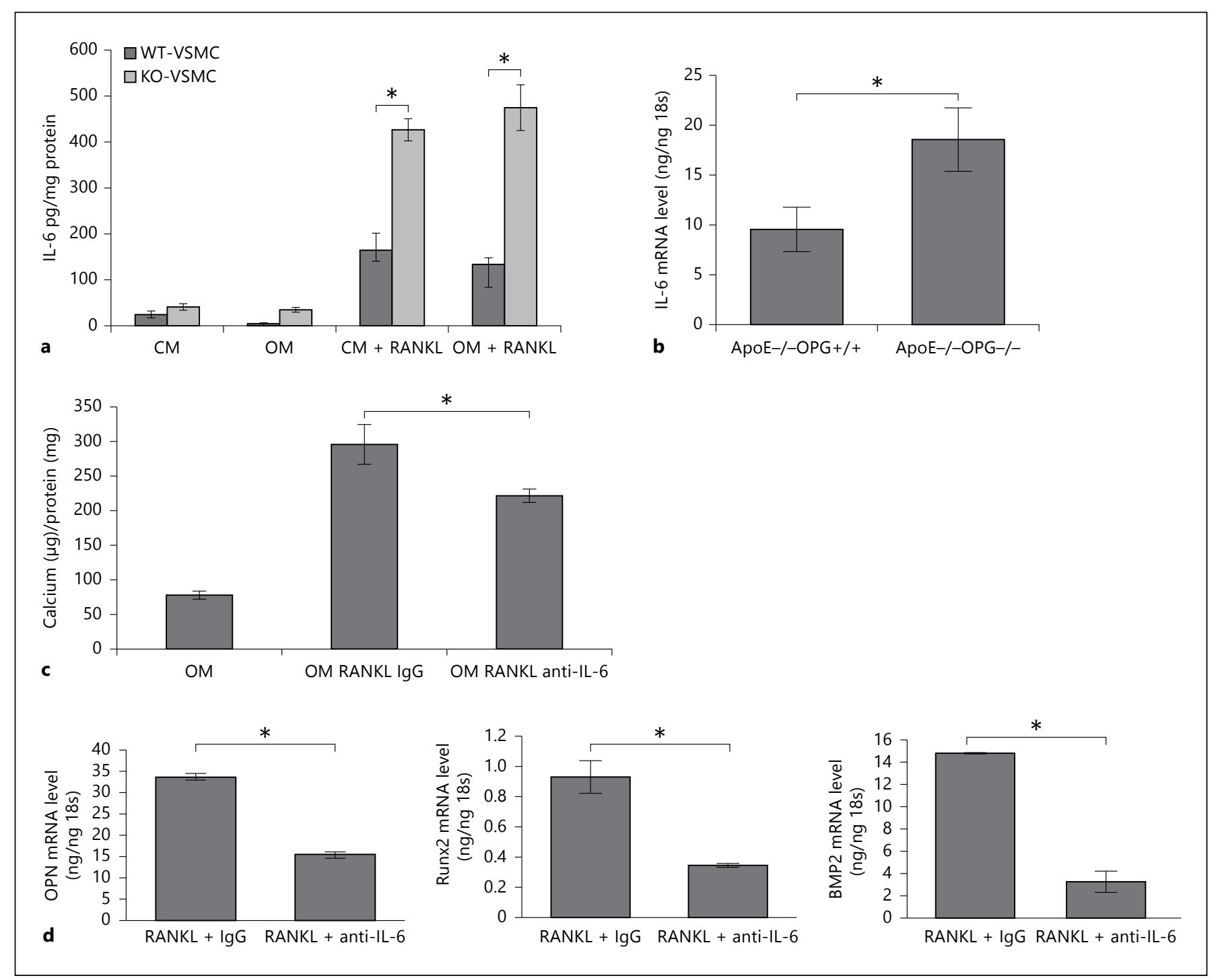

Fig. 6. RANKL induces upregulation of IL-6. a ELISA with cell supernatant from WT-VSMCs and KO-VSMCs treated with RANKL in $\mathrm{CM}$ and $\mathrm{OM}$ conditions. ${ }^{*} \mathrm{p}<0.05 \mathrm{KO}$-VSMC RANKL vs. WTVSMC RANKL. b qPCR for IL-6 expression in aortas freshly isolated from 12-week-old ApoE-/-OPG-/- and ApoE-/-OPG+/+ mice. c Calcification assay with KO-VSMCs cultured in OM, treat- ed RANKL and isotype control antibody (OM RANKL IgG), and treated RANKL and anti-IL-6 neutralizing antibody (OM RANKL anti-IL-6) for 7 days. ${ }^{*} \mathrm{p}<0.05$ OM RANKL IgG vs. OM RANKL anti-IL-6. d qPCR for OPN, Runx2 and BMP2 of KO-VSMCs treated with RANKL or RANKL and anti-IL- 6 antibody for 4 days. $* \mathrm{p}<0.05$. suggesting that hypercholesterolemia and a lack of OPG induce VSMC osteochondrogenic differentiation. In addition, in vitro KO-VSMCs appeared to express similar levels of smooth muscle markers when freshly isolated; however, with time in culture, we observed downregulation of SMa-actin, enhanced ERK phosphorylation and enhanced Runx2 activity, suggesting that KO-VSMCs were undergoing a phenotypic change even before the induction of mineralization. Whether unabated RANKL is responsible for these observations is a critical question. As reported by many, RANKL is expressed in the vessel wall $[5,11,12,35]$. Accordingly, we have found that RANKL was associated with medial calcium deposits and VSMCs in young ApoE-/-OPG-/- mice (fig. 5e). In vivo inhibition of RANKL with an anti-human RANKL antibody (denosumab) in a transgenic mouse model challenged with prednisolone appeared to inhibit vascular calcification [36]. These findings strongly suggest a 
Fig. 7. Proposed model for RANKL-mediated vascular calcification. In the absence of OPG, RANKL in an IL-6-dependent and IL-6-independent manner upregulates a subset of osteochondrogenic genes (BMP2, Runx 2 and OPN) and in an IL-6-independent manner downregulates MGP. The combination of these effects leads to increased vascular calcification. Furthermore, IL-6 induces RANKL and OPG, possibly suggesting a feed-forward process.

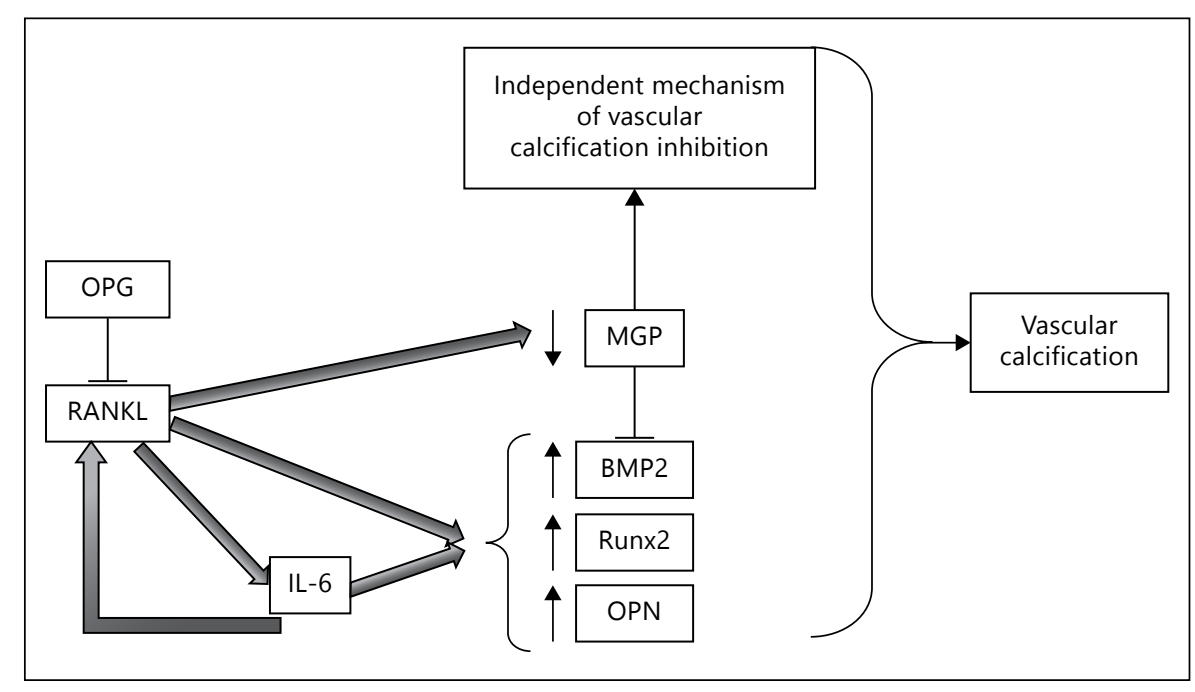

role for RANKL in vascular calcification. However, in vitro data on isolated SMCs have not always supported this hypothesis. A number of studies suggest that RANKL is a positive regulator of SMC mineralization, but others dispute these findings [35, 37-40]. Correspondingly, we found that RANKL did not increase WT-VSMCs calcification in response to osteogenic conditions (of note: WT-VSMCs secreted very high levels of OPG; fig. 2). However, we found that KO-VSMCs deposited more calcium than WT-VSMCs, which correlated with elevated osteochondrogenic genes (fig. 3). We further found that inhibition of RANKL signaling, by downregulation of its cell surface receptor RANK, led to the rescue of mineralization in KO-VSMCs as did OPG re-expression, which importantly also led to osteochondrogenic phenotype normalization (fig. 4; online suppl. fig. 5). In addition, RANKL accelerated osteochondrogenic phenotypic transition and was a potent inducer of calcification in KO-VSMCs (fig. 5; online suppl. fig. 7). These latter observations are in agreement with and expand an observation by Osako et al. [35] and Panizo et al. [37]. Taken together, these results suggest that RANKL is an inducer of BMP type factors that in turn activate Runx2 and osteochondrogenic genes. At the same time, RANKL is an inhibitor of MGP expression that is a potent BMP inhibitor and aids in matrix vesicle clearance $[3,26,41,42]$. Interestingly, even if RANKL induced gene changes and Runx2 activation, it was not sufficient to induce VSMC mineralization in control conditions, suggesting that the mineralizing-permissive conditions mimicked in vitro by the OM are necessary for RANKL to act as an enhancer of VSMC calcification.

Calcification in OPG-Deficient VSMCs
We also show that RANKL is a potent inducer of IL-6 in VSMCs (fig. 6). We have recently found that IL-6 had no effect on WT-VSMC calcification but did upregulate OPN and downregulate MGP expression [25]. However, in KO-VSMCs, IL-6 weakly induced mineralization and partially mediated the RANKL induction of mineralization and the expression of OPN, Runx2 and BMP2, but not MGP (online suppl. fig. 8; fig. 6). Yao et al. [26] have shown that IL- 6 modulates vascular calcification by affecting the ability of MGP to bind and inactivate BMP2. To our knowledge, this is the first report that establishes IL-6 as a direct regulator of VSMC mineralization and mineralization genes. IL- 6 is a CVD biomarker and a strong predictor of coronary artery disease and sudden cardiovascular death; however, the mechanism of this cytokine modulation of CVD and vascular calcification is still controversial [43-45]. Some experimental studies suggest that IL-6 is proatherogenic while others indicate that physiological levels of IL- 6 are necessary to maintain the vascular inflammatory response in check [45-50]. While vascular calcification was not evaluated in any of these studies, it is clinically well-established that IL-6 is an independent predictor of cardiovascular events in chronic kidney disease patients who are affected by extensive vascular calcification [51].

In conclusion, we have provided evidence that, in the setting of OPG deficiency and in an osteogenic environment, RANKL accelerated VSMC calcification. Furthermore, we have presented evidence that RANKL accelerated the osteochondrogenic conversion of VSMCs partially via the induction of IL- 6 . Thus, we propose a model whereby unopposed RANKL in an IL-6-independent and IL-6-de- 
pendent manner upregulates a subset of osteochondrogenic genes (BMP2, Runx2 and OPN) and in an IL-6-independent manner downregulates MGP. In addition, the observed induction of RANKL, RANK and OPG by IL-6 in VSMCs may indicate a feed-forward loop that further propagates VSMC mineralization. The combination of these effects leads to increased vascular calcification in mineralizing-permissive conditions (fig. 7). Given the epidemiological data in humans correlating the OPG/RANKL system with CVD and vascular calcification, targeting the RANKL/ RANK system in VSMCs may be a therapeutic approach for reducing the vascular calcification burden in CVD.

\section{Acknowledgments}

This work has been supported by funding from $\mathrm{NIH}$ R01HL093469-01 and R01DK094434-01.

\section{References}

$\checkmark 1$ Sage AP, Tintut Y, Demer LL: Regulatory mechanisms in vascular calcification. Nat Rev Cardiol 2010;7:528-536.

-2 Moe SM, Reslerova M, Ketteler M, O'neill K, Duan D, Koczman J, Westenfeld R, JahnenDechent W, Chen NX: Role of calcification inhibitors in the pathogenesis of vascular calcification in chronic kidney disease (CKD). Kidney Int 2005;67:2295-2304.

-3 Shao JS, Cheng SL, Sadhu J, Towler DA: Inflammation and the osteogenic regulation of vascular calcification: a review and perspective. Hypertension 2010;55:579-592.

4 Abedin M, Tintut Y, Demer LL: Vascular calcification: mechanisms and clinical ramifications. Arterioscler Thromb Vasc Biol 2004;24: 1161-1170.

-5 Rattazzi M, Bennett BJ, Bea F, Kirk EA, Ricks JL, Speer M, Schwartz SM, Giachelli CM, Rosenfeld ME: Calcification of advanced atherosclerotic lesions in the innominate arteries of ApoE-deficient mice: potential role of chondrocyte-like cells. Arterioscler Thromb Vasc Biol 2005;25:1420-1425.

6 Lau WL, Pai A, Moe SM, Giachelli CM: Direct effects of phosphate on vascular cell function. Adv Chronic Kidney Dis 2011;18:105-112.

7 Lau WL, Festing MH, Giachelli CM: Phosphate and vascular calcification: emerging role of the sodium-dependent phosphate cotransporter PiT-1. Thromb Haemost 2010; 104:464-470.

8 Lacey DL, Timms E, Tan HL, Kelley MJ, Dunstan CR, Burgess T, Elliott R, Colombero A, Elliott G, Scully S, Hsu H, Sullivan J, Hawkins N, Davy E, Capparelli C, Eli A, Qian YX, Kaufman S, Sarosi I, Shalhoub V, Senaldi G, Guo J, Delaney J, Boyle WJ: Osteoprotegerin ligand is a cytokine that regulates osteoclast differentiation and activation. Cell 1998;93: 165-176.

\$ Yun TJ, Chaudhary PM, Shu GL, Frazer JK, Ewings MK, Schwartz SM, Pascual V, Hood LE, Clark EA: OPG/FDCR-1, a TNF receptor family member, is expressed in lymphoid cells and is up-regulated by ligating CD40. J Immunol 1998;161:6113-6121.

$\checkmark 10$ Yun TJ, Tallquist MD, Aicher A, Rafferty KL, Marshall AJ, Moon JJ, Ewings ME, Mohaupt
M, Herring SW, Clark EA: Osteoprotegerin, a crucial regulator of bone metabolism, also regulates $B$ cell development and function. J Immunol 2001;166:1482-1491.

11 Sandberg WJ, Yndestad A, Øie E, Smith C, Ueland T, Ovchinnikova O, Robertson AK, Müller F, Semb AG, Scholz H, Andreassen AK, Gullestad L, Damås JK, Frøland SS, Hansson GK, Halvorsen B, Aukrust P: Enhanced T-cell expression of rank ligand in acute coronary syndrome: possible role in plaque destabilization. Arterioscler Thromb Vasc Biol 2006;26:857-863.

$\checkmark 12$ Dhore CR, Cleutjens JP, Lutgens E, Cleutjens KB, Geusens PP, Kitslaar PJ, Tordoir JH, Spronk HM, Vermeer C, Daemen MJ: Differential expression of bone matrix regulatory proteins in human atherosclerotic plaques. Arterioscler Thromb Vasc Biol 2001;21: 1998-2003.

13 Bennett BJ, Scatena M, Kirk EA, Rattazzi M, Varon RM, Averill M, Schwartz SM, Giachelli CM, Rosenfeld ME: Osteoprotegerin inactivation accelerates advanced atherosclerotic lesion progression and calcification in older ApoE-/- mice. Arterioscler Thromb Vasc Biol 2006;26:2117-2124.

$\checkmark 14$ Callegari A, Coons M, Ricks JL, Yang HL, Gross TS, Huber P, Rosenfeld ME, Scatena M: Bone marrow- or vessel wall-derived osteoprotegerin is sufficient to reduce atherosclerotic lesion size and vascular calcification. Arterioscler Thromb Vasc Biol 2013;33:24912500.

15 Morony S, Tintut Y, Zhang Z, Cattley RC, Van G, Dwyer D, Stolina M, Kostenuik PJ, Demer LL: Osteoprotegerin inhibits vascular calcification without affecting atherosclerosis in LDLR(-/-) mice. Circulation 2008; 117: 411-420.

16 Malyankar UM, Scatena M, Suchland KL, Yun TJ, Clark EA, Giachelli CM: Osteoprotegerin is an alpha vbeta 3-induced, NF-kappa B-dependent survival factor for endothelial cells. J Biol Chem 2000;275:2095920962.

17 Hofbauer LC: Pathophysiology of rank ligand (RANKL) and osteoprotegerin (OPG). Ann Endocrinol (Paris) 2006;67:139-141.
18 Schoppet M, Sattler AM, Schaefer JR, Herzum M, Maisch B, Hofbauer LC: Increased osteoprotegerin serum levels in men with coronary artery disease. J Clin Endocrinol Metab 2003; 88:1024-1028.

19 Jono S, Otsuki S, Higashikuni Y, Shioi A, Mori K, Hara K, Hashimoto H, Ikari Y: Serum osteoprotegerin levels and long-term prognosis in subjects with stable coronary artery disease. J Thromb Haemost 2010;8: 1170-1175.

20 Rice J, Courter DL, Giachelli CM, Scatena M: Molecular mediators of alphavbeta3-induced endothelial cell survival. J Vasc Res 2006;43: 422-436.

21 Sangiorgi G, Rumberger JA, Severson A, Edwards WD, Gregoire J, Fitzpatrick LA, Schwartz RS: Arterial calcification and not lumen stenosis is highly correlated with atherosclerotic plaque burden in humans: a histologic study of 723 coronary artery segments using nondecalcifying methodology. J Am Coll Cardiol 1998;31:126-133.

22 Ge C, Xiao G, Jiang D, Yang Q, Hatch NE, Roca $\mathrm{H}$, Franceschi RT: Identification and functional characterization of ERK/MAPK phosphorylation sites in the Runx2 transcription factor. J Biol Chem 2009;284:3253332543.

23 Johnson KA, Polewski M, Terkeltaub RA: Transglutaminase 2 is central to induction of the arterial calcification program by smooth muscle cells. Circ Res 2008;102:529-537.

24 Speer MY, Li X, Hiremath PG, Giachelli CM: Runx2/Cbfa1, but not loss of myocardin, is required for smooth muscle cell lineage reprogramming toward osteochondrogenesis. J Cell Biochem 2010;110:935-947.

-25 Deuell KA, Callegari A, Giachelli CM, Rosenfeld ME, Scatena M: RANKL enhances macrophage paracrine pro-calcific activity in high phosphate-treated smooth muscle cells: dependence on IL- 6 and TNF- $\alpha$. J Vasc Res 2012;49:510-521.

26 Yao Y, Watson AD, Ji S, Boström KI: Heat shock protein 70 enhances vascular bone morphogenetic protein-4 signaling by binding matrix GLA protein. Circ Res 2009;105: 575-584. 
27 Di Bartolo BA, Schoppet M, Mattar MZ, 36 Helas S, Goettsch C, Schoppet M, Zeitz U, Rachner TD, Shanahan CM, Kavurma MM: Calcium and osteoprotegerin regulate IGF1R expression to inhibit vascular calcification. Cardiovasc Res 2011;91:537-545.

28 Kiechl S, Schett G, Schwaiger J, Seppi K, Eder P, Egger G, Santer P, Mayr A, Xu Q, Willeit J: Soluble receptor activator of nuclear factor-kappa B ligand and risk for cardiovascular disease. Circulation 2007;116: 385-391.

29 Jørgensen L, Hansen JB, Brox J, Mathiesen E, Vik A, Jacobsen BK: Serum osteoprotegerin levels are related to height loss: the Tromsø study. Eur J Epidemiol 2011;26:305-312.

-30 Ueland T, Yndestad A, Øie E, Florholmen G, Halvorsen B, Frøland SS, Simonsen S, Christensen G, Gullestad L, Aukrust P: Dysregulated osteoprotegerin/RANK ligand/RANK axis in clinical and experimental heart failure. Circulation 2005;111:2461-2468.

-31 Fahrleitner-Pammer A, Dobnig H, Dimai $\mathrm{HP}$, Holzer H, Benesch T, Borchhardt K, Cejka D, Haas M: The effect of RANKL and OPG on bone mineral density in pre-dialysis chronic renal failure. Clin Nephrol 2009;71: 652-659.

32 Kiechl S, Schett G, Wenning G, Redlich K, Oberhollenzer M, Mayr A, Santer P, Smolen J, Poewe W, Willeit J: Osteoprotegerin is a risk factor for progressive atherosclerosis and cardiovascular disease. Circulation 2004;109: 2175-2180.

33 Hosbond SE, Poulsen TS, Diederichsen AC, Nybo M, Rasmussen LM, Mickley H: Osteoprotegerin as a marker of atherosclerosis: a systematic update. Scand Cardiovasc J 2012; 46:203-211.

-34 Jono S, Ikari Y, Shioi A, Mori K, Miki T, Hara K, Nishizawa Y: Serum osteoprotegerin levels are associated with the presence and severity of coronary artery disease. Circulation 2002; 106:1192-1194.

35 Osako MK, Nakagami H, Koibuchi N, Shimizu H, Nakagami F, Koriyama H, Shimamura M, Miyake T, Rakugi H, Morishita R: Estrogen inhibits vascular calcification via vascular RANKL system: common mechanism of osteoporosis and vascular calcification. Circ Res 2010;107:466-475. Hempel U, Morawietz H, Kostenuik PJ, Erben RG, Hofbauer LC: Inhibition of receptor activator of NF-kappaB ligand by denosumab attenuates vascular calcium deposition in mice. Am J Pathol 2009; 175:473-478.

37 Panizo S, Cardus A, Encinas M, Parisi E, Valcheva P, López-Ongil S, Coll B, Fernandez E, Valdivielso JM: RANKL increases vascular smooth muscle cell calcification through a RANK-BMP4-dependent pathway. Circ Res 2009;104:1041-1048.

38 Tseng W, Graham LS, Geng Y, Reddy A, Lu J, Effros RB, Demer L, Tintut Y: PKA-induced receptor activator of NF-kappab ligand (RANKL) expression in vascular cells mediates osteoclastogenesis but not matrix calcification. J Biol Chem 2010;285:29925-29931.

39 Byon CH, Sun Y, Chen J, Yuan K, Mao X, Heath JM, Anderson PG, Tintut Y, Demer LL, Wang D, Chen Y: Runx2-upregulated receptor activator of nuclear factor $\mathrm{\kappa B}$ ligand in calcifying smooth muscle cells promotes migration and osteoclastic differentiation of macrophages. Arterioscler Thromb Vasc Biol 2011; 31:1387-1396.

40 Olesen M, Skov V, Mechta M, Mumm BH, Rasmussen LM: No influence of OPG and its ligands, RANKL and TRAIL, on proliferation and regulation of the calcification process in primary human vascular smooth muscle cells. Mol Cell Endocrinol 2012;362:149-156.

-41 Speer MY, McKee MD, Guldberg RE, Liaw L, Yang HY, Tung E, Karsenty G, Giachelli CM: Inactivation of the osteopontin gene enhances vascular calcification of matrix GLA protein-deficient mice: evidence for osteopontin as an inducible inhibitor of vascular calcification in vivo. J Exp Med 2002;196:1047-1055.

42 Steitz SA, Speer MY, Curinga G, Yang HY, Haynes P, Aebersold R, Schinke T, Karsenty G, Giachelli CM: Smooth muscle cell phenotypic transition associated with calcification: Upregulation of Cbfa1 and downregulation of smooth muscle lineage markers. Circ Res 2001;89:1147-1154.

43 Murabito JM, Keyes MJ, Guo CY, Keaney JF, Vasan RS, D'Agostino RB, Benjamin EJ: Cross-sectional relations of multiple inflammatory biomarkers to peripheral arterial disease: The Framingham Offspring Study. Atherosclerosis 2009;203:509-514.
44 Tzoulaki I, Murray GD, Lee AJ, Rumley A, Lowe GD, Fowkes FG: C-reactive protein, interleukin-6, and soluble adhesion molecules as predictors of progressive peripheral atherosclerosis in the general population: Edinburgh Artery Study. Circulation 2005;112: 976-983.

45 Empana JP, Jouven X, Canouï-Poitrine F, Luc G, Tafflet M, Haas B, Arveiler D, Ferrieres J, Ruidavets JB, Montaye M, Yarnell J, Morange P, Kee F, Evans A, Amouyel P, Ducimetiere P: C-reactive protein, interleukin 6 , fibrinogen and risk of sudden death in European middleaged men: the prime study. Arterioscler Thromb Vasc Biol 2010;30:2047-2052.

46 Huber SA, Sakkinen P, Conze D, Hardin N, Tracy R: Interleukin-6 exacerbates early atherosclerosis in mice. Arterioscler Thromb Vasc Biol 1999;19:2364-2367.

47 Schuett H, Oestreich R, Waetzig GH, Annema W, Luchtefeld M, Hillmer A, Bavendiek U, von Felden J, Divchev D, Kempf T, Wollert KC, Seegert D, Rose-John S, Tietge UJ, Schieffer B, Grote K: Transsignaling of interleukin-6 crucially contributes to atherosclerosis in mice. Arterioscler Thromb Vasc Biol 2012; 32:281-290.

48 Elhage R, Clamens S, Besnard S, Mallat Z, Tedgui A, Arnal J, Maret A, Bayard F: Involvement of interleukin- 6 in atherosclerosis but not in the prevention of fatty streak formation by 17beta-estradiol in apolipoprotein E-deficient mice. Atherosclerosis 2001;156: 315-320.

49 Madan M, Bishayi B, Hoge M, Amar S: Atheroprotective role of interleukin-6 in dietand/or pathogen-associated atherosclerosis using an ApoE heterozygote murine model. Atherosclerosis 2008;197:504-514.

50 Schieffer B, Selle T, Hilfiker A, Hilfiker-Kleiner D, Grote K, Tietge UJ, Trautwein C, Luchtefeld M, Schmittkamp C, Heeneman S, Daemen MJ, Drexler H: Impact of interleukin-6 on plaque development and morphology in experimental atherosclerosis. Circulation 2004;110:3493-3500.

51 Okamura DM, Himmelfarb J: Tipping the redox balance of oxidative stress in fibrogenic pathways in chronic kidney disease. Pediatr Nephrol 2009;24:2309-2319. 